\title{
REVIEWS
}

\section{Mobile Health for Arrhythmia Diagnosis and Management}

\author{
Jayson R. Baman, $\mathrm{MD}^{7}$ (D), Daniel T. Mathew, $B A^{2}$, Michael Jiang, $M D^{7}$, and \\ Rod S. Passman, MD, MSCE ${ }^{1,2,3}$
}

\begin{abstract}
'Division of Cardiology, Department of Medicine, Northwestern University Feinberg School of Medicine, Chicago, IL, USA; ${ }^{2}$ Northwestern University Feinberg School of Medicine, Chicago, IL, USA; ${ }^{3}$ Center for Arrhythmia Research, Northwestern University Feinberg School of Medicine, Chicago, IL, USA.
\end{abstract}

Palpitations are a common symptom managed by general practitioners and cardiologists; atrial fibrillation $(\mathrm{AF})$ is the most common arrhythmia in adults. The recent commercial availability of smartphone-based devices and wearable technologies with arrhythmia detection capabilities has revolutionized the diagnosis and management of these common medical issues, as it has placed the power of arrhythmia detection into the hands of the patient. Numerous mobile health (mHealth) devices that can detect, record, and automatically interpret irregularities in heart rhythm and abrupt changes in heart rate using photoplethysmography (PPG)- and electrocardiogrambased technologies are now commercially available. As opposed to prescription-based external rhythm monitoring approaches, these devices are more inexpensive and allow for longer-term monitoring, thus increasing sensitivity for arrhythmia detection, particularly for patients with infrequent symptoms possibly due to cardiac arrhythmias. These devices can be used to correlate symptoms with cardiac arrhythmias, assess efficacy and toxicities of arrhythmia therapies, and screen the population for serious rhythm disturbances such as AF. Although several devices have received clearance for $\mathrm{AF}$ detection from the United States Food \& Drug Administration, limitations include the need for ECG confirmation for arrhythmias detected by PPG alone, false positives, false negatives, charging requirements for the battery, and financial cost. In summary, the growth of commercially available devices for remote, patient-facing rhythm monitoring represents an exciting new opportunity in the care of patients with palpitations and known or suspected dysrhythmias. Physicians should be familiar with the evidence that underlies their added value to patient care and, importantly, their current limitations.

KEYWORDS: Arrhythmia; Palpitations; Technology; Atrial fibrillation.

J Gen Intern Med 37(1):188-97

DOI: $10.1007 /$ s11606-021-07007-w

(C) Society of General Internal Medicine 2021

\section{INTRODUCTION}

Palpitations are a common complaint and atrial fibrillation (AF) is the most common sustained arrhythmia in adults. Most

Received January 6, 2021

Accepted June 25, 2021

Published online July 19, 2021 palpitations occur during normal sinus rhythm and $\mathrm{AF}$ can be the cause of palpitations; however, it is often asymptomatic. In contrast to the majority of palpitations which are benign in nature, AF is associated with increased risk for thromboembolic events, particularly cardioembolic ischemic strokes, whether or not they are associated symptoms. ${ }^{1,2}$ In addition, $\mathrm{AF}$ has been recognized as a contributor to other conditions, such as dementia, heart failure, and all-cause mortality. ${ }^{3,4}$ ECG confirmation is necessary for the diagnosis of all cardiac arrhythmias in order to assess severity, implications, and treatment options. Unfortunately, for those with infrequent and short-lived symptoms and those with paroxysmal AF with or without symptoms, traditional short-term cardiac monitors are ill-suited to provide this critical information. However, the recent commercial availability of smartphone-based devices and wearable technologies with arrhythmia detecting capabilities has now placed the power of arrhythmia detection into the hands of the patient. As a result, clinicians in all specialties - from general practitioners to cardiac electrophysiologists - are faced with new diagnostic, and thus management, opportunities, and challenges. How does one incorporate these technologies into clinical practice? Can we trust the information from these devices? Are confirmatory tests required? What is our responsibility to respond to a test that we did not order? Here, we explore the landscape of commercially available wearable technologies and smartphone-related devices, henceforth described as "mobile health," or mHealth; the utility and limitations of the data gathered; and the associated regulatory and legal implications.

Historically, pulse palpation at bedside was the first clinical tool used in the detection of arrhythmias. The invention of the electrocardiogram (ECG) by Einthoven in 1895 allowed for a more nuanced understanding of rhythm disturbances. Physical exam and ECG, unfortunately, provide only snapshots of a patient's heart rhythm and thus are insufficient for correlating symptoms with possible rhythm disturbances if they occurred infrequently or for identifying occult paroxysmal dysrhythmias. ${ }^{5}$ In fact, in the early studies that form our foundation for our current understanding of $\mathrm{AF}$, the recognition of $\mathrm{AF}$ was limited to scenarios where a patient was symptomatic and sought care, at which time electrocardiogram confirmed AF, or where a patient was asymptomatic at the time of a medical 
encounter but found to be in AF on electrocardiogram that was obtained in response to an abnormal physical examination. ${ }^{6,7}$

To improve duration of surveillance and sensitivity of arrhythmia screening, provider-initiated wearable screening tools became increasingly common. ${ }^{8}$ In parallel, for the past few decades, commercially available fitness trackers have become more popular. In some cases, patients reported inappropriate tachycardia during periods of rest, prompting medical attention that confirmed true arrhythmia. This observation prompted manufacturers to incorporate specific algorithms into their devices capable of detecting not only rapid rates, but also irregular rhythms regardless of heart rate. With continued technological advancements, the field of patientinitiated heart rate and rhythm monitors has evolved into a meaningful force in clinical care, with significant implications for dysrhythmia identification and management.

\section{LIMITATIONS OF TRADITIONAL CARDIAC MONITORING FOR PALPITATIONS AND AF}

The conventional approach to ambulatory rhythm monitoring for palpitations and AF has centered on prescription-based devices and strategies, including the Holter monitor, event monitor, event/patch monitor, and, in some cases, implantable cardiac monitors (ICM). All of these devices are considered in the armamentarium of ambulatory rhythm monitors for the following situations ${ }^{1}$ : recurrent palpitations ${ }^{2}$; recurrent syncope, for which dysrhythmia is considered a contributing cause ${ }^{3}$; stroke or suspected cardioembolic event, where occult AF could be a causal factor ${ }^{4}$; evaluation of the burden or rate control of AF; and ${ }^{5}$ survey for adverse effects of medications. This list is not exhaustive, but it underscores the utility of remote rhythm monitoring, where data collected over a long period of time can inform clinical decisions and augment data collected during smaller "snapshots" in time, such as an outpatient appointment or inpatient hospital stay.

For patients experiencing and practitioners evaluating palpitations, the on-demand electrocardiographic tracings offered by many commercially available mHealth technologies are attractive. Less than half of patients presenting to the emergency department with palpitations are given a cardiac diagnosis ${ }^{9,10}$; however, this figure may underestimate the true burden given the limited time of screening. In the diagnostic evaluation of palpitations, the data have shown that long durations of rhythm surveillance are most effective in identifying an arrhythmic cause, especially in patients whose symptoms are sporadic. The diagnostic value of a 30-day ambulatory rhythm monitoring in a prospective study of patients with palpitations, syncope, or presyncope was $27.8 \%$, only marginally higher than a study of 24-h Holter monitoring in patients with palpitations and altered consciousness (15.8\%); this low yield for arrhythmia detection has been replicated in other studies. ${ }^{1-13}$ Studies have shown that ambulatory rhythm monitors most frequently capture benign ventricular or atrial ectopy, supraventricular tachycardias, or sinus rhythm during complaints of palpitations. ${ }^{13,14}$

In general, use of these devices can be limited by the need for physician orders, financial costs to the patient, narrow time of rhythm surveillance, and patient compliance that is inversely proportional to the length of prescribed monitoring. Although the implantable loop recorder has been shown to be superior to traditional Holter monitors for identifying AF, this strategy is suboptimal given its invasive nature and cost, and it may be unnecessary in all but those at highest risk for stroke where the diagnosis of AF would change management. ${ }^{15,16}$ Multiple prior studies have suggested that continuous, longterm monitoring is superior to intermittent rhythm monitoring for detecting AF events, given the often paroxysmal nature of AF. ${ }^{17-19}$ The burgeoning data suggesting the superior sensitivity of continuous monitoring, as well as the increasing importance of providing this monitoring in an inexpensive, accessible manner, have provided a ripe environment for commercially available mobile health technologies.

\section{COMMERCIALLY AVAILABLE MHEALTH TECHNOLOGY}

mHealth devices that are capable of heart rate and/or heart rhythm monitoring have taken on various forms, including smartphone apps, wrist-worn smartwatches, rings, necklaces, clothing-embedded sensors, and patches ${ }^{5,20}$ (Fig. 1; Table 2). mHealth technologies for detecting arrhythmias can be divided into two broad categories: ECG-based tracings and nonECG-based modalities.

The primary non-ECG-based modality utilized by mHealth for arrhythmia detection is photoplethysmography (PPG). Devices utilize a light source and a photodetector to determine changes in light intensity on the skin surface, correlating to changes in blood tissue volume during different phases of the cardiac cycle. Algorithms for AF detection were developed after it was noted that the PPG designed for heart rate (HR) assessment during exercise alerted some individuals of high heart rates even when sedentary, prompting further evaluation. Varying pulse wave intensities and intervals can signal an inconsistent stroke volume and irregular heartbeat, respectively, both of which are characteristic findings in AF. ${ }^{27}$ Studies evaluating the accuracy of heart rate detection via PPG technology compared to ECG have shown the correlation of heart rate assessments within just several beats per minute; this suggests that if these devices were to capture abrupt changes in heart rate, such that would indicate a sudden change in rhythm, these findings could be reliable (Fig. 2A). ${ }^{28}$ Though ECG remains the gold standard for AF diagnosis, PPG is already present in most commercially available smartphone and wearable mobile devices and thus offers a low-cost method for arrhythmia surveillance if not allowing for specific arrhythmia diagnosis. Arrhythmias detected by PPG alone should be confirmed by further ECG assessment. 
A

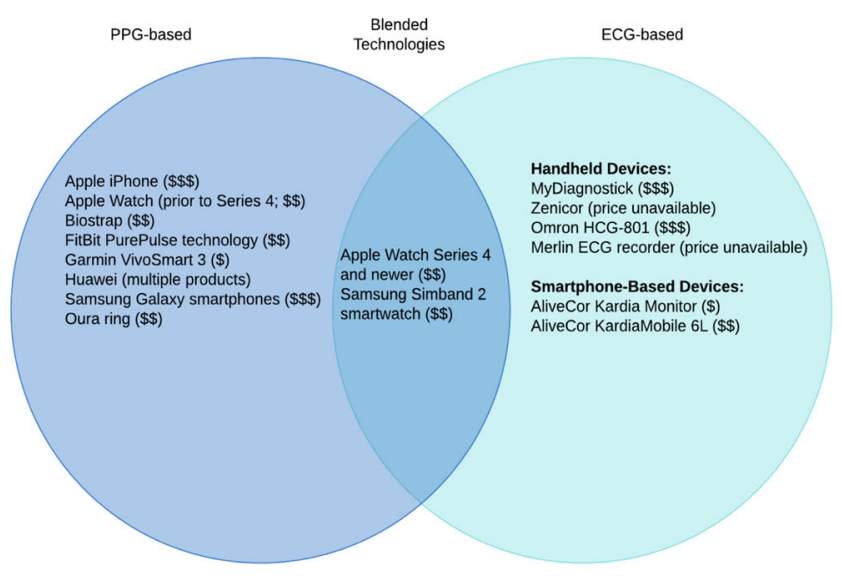

B $\$ \$$ represents $>\$ 500$ $\$ \$$ represents $\$ 100$ to $\$ 500$
$\$$ represents $<\$ 100$
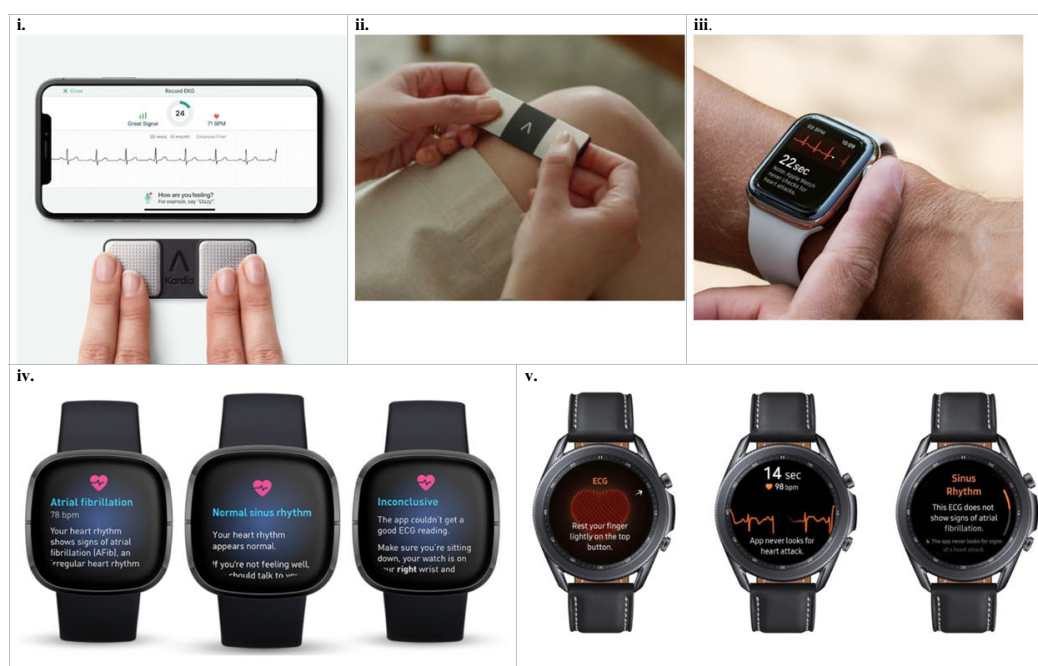

(i)

AliveCor Kardia Monitor device series. This device utilizes a single-lead ECG strip that can be mounted onto the back of a phone case or external card. Patients place one finger from each hand on to the two electrodes on the strip, and the devices records a single lead ECG tracing. These devices have integrated algorithms that are able to detect the presence of AF based on the irregularity of the RR intervals and presence of a P-wave. Picture source: https://store.alivecor.com/products/kardiamobile

(ii) AliveCor KardiaMobile 6L. This device produces a six-lead ECG tracing by using a sensor on the back of the device that can be better visualized in lead II. Picture soper

Den well studied for the detection of AF. Earlier iterations of the watch relied solely on PPG to detect heart rhythm irregularities, however the Series 4 and more recent iterations provide blended PPG and ECG capabilities. Picture source: https://www.apple.com/healthcare/apple-watch/

(iv) Fitbit Sense. This wearable device uses PPG technology to detect heart rhythm abnormalities; there are additional apps available to provide ECG tracings. In this picture, we see that the Fitbit Sense describes the heart rhythm as AF, normal sinus or inconclusive. provide ECG tracings. In this picture, we see that the Fitbit Sense
Picture source: https://www.fitbit.com/global/us/technology/ecg

(v) Samsung Galaxy Watch. This series of wearable devices can detect irregular heart rhythms with blended PPG and ECG technology. Here, we see multiple screens showing the device's prompt for the user to participate in an ECG measurement, a live tracing and an interpreted rhythm as sinus, respectively. Picture source: https://www.cnet.com/how-to/samsung-adds-ecg-monitor-to-galaxy-watch-3and-galaxy-watch-active-2/

Figure 1 (A) Venn diagram depicting the primary mechanism of arrhythmia detection for select commercially available devices. Estimated retail price included. (B) Images of selective devices. (i) AliveCor Kardia Monitor device series. This device utilizes a single-lead ECG strip that can be mounted onto the back of a phone case or external card. Patients place one finger from each hand on to the two electrodes on the strip, and the devices records a single-lead ECG tracing. These devices have integrated algorithms that are able to detect the presence of AF based on the irregularity of the RR intervals and presence of a P-wave. Picture source: https://store.alivecor.com/products/kardiamobile, (ii) AliveCor KardiaMobile 6L. This device produces a six-lead ECG tracing by using a sensor on the back of the device that can be placed on the leg. It may be well suited for patients with atrial flutter, where flutter waves are better visualized in lead II. Picture source: https://store.alivecor.com/ products/kardiamobile6l, (iii) Apple Watch. This series of wearable devices has been well studied for the detection of AF. Earlier iterations of the watch relied solely on PPG to detect heart rhythm irregularities, however the Series $\mathbf{4}$ and more recent iterations provide blended PPG and ECG capabilities. Picture source: https://www.apple.com/healthcare/apple-watch/, (iv) Fitbit Sense. This wearable device uses PPG technology to detect heart rhythm abnormalities; there are additional apps available to provide ECG tracings. In this picture, we see that the Fitbit Sense describes the heart rhythm as AF, normal sinus or inconclusive. Picture source: https://www.fitbit.com/global/us/technology/ecg, (v) Samsung

Galaxy Watch. This series of wearable devices can detect irregular heart rhythms with blended PPG and ECG technology. Here, we see multiple screens showing the device's prompt for the user to participate in an ECG measurement, a live tracing and an interpreted rhythm as sinus, respectively. Picture source: https://www.cnet.com/how-to/samsung-adds-ecg-monitor-to-galaxy-watch-3-and-galaxy-watch-active-2/ 
(A)

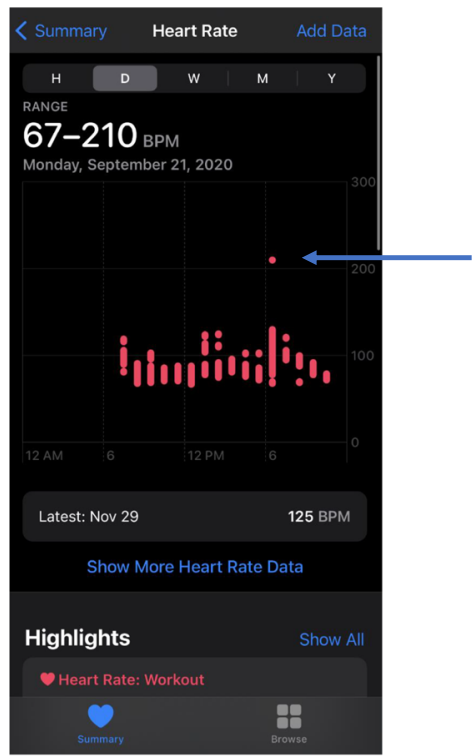

(B)

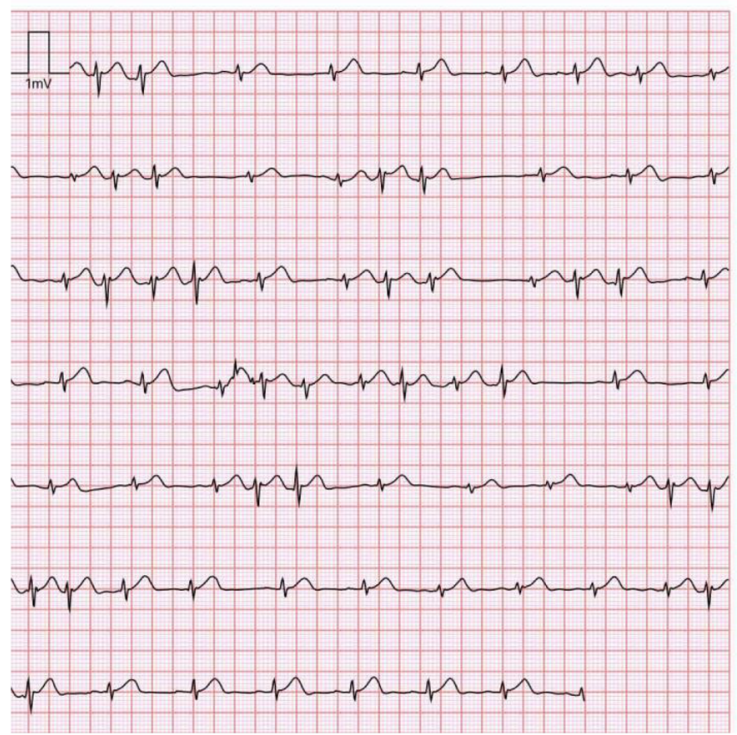

(C)
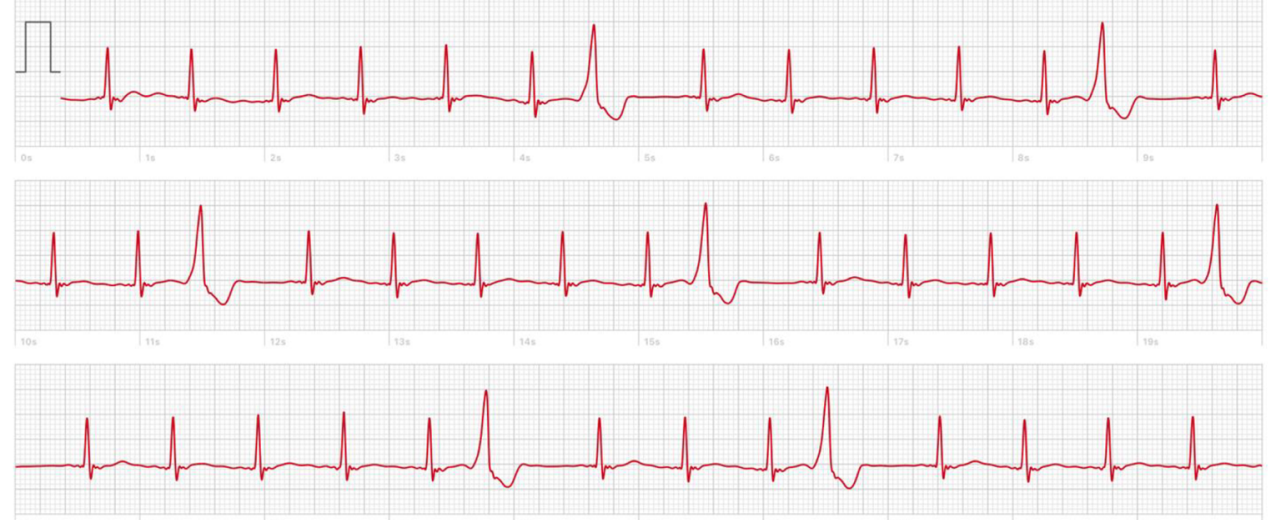

Figure 2 Data obtained from three patients using the Apple Watch. (A) PPG data from the Apple Watch showed an abrupt increase in heart rate (blue arrow) that was associated with palpitations. Subsequent prescription-based ambulatory monitoring revealed paroxysmal supraventricular tachycardia with pre-excitation; the patient underwent successful ablation for Wolff-Parkinson-White syndrome. In panels (B) and (C), ECG-rendered from the Apple Watch captured premature atrial complexes and premature ventricular complexes, respectively, that corresponded to the times of the patients' symptoms.

\section{ARRHYTHMIA DETECTION TECHNOLOGY AND DIAGNOSTIC ACCURACY}

The major devices and technologies using PPG, ECG, and blended technologies are shown in Figure 1. The field of ambulatory, smartphone-based PPG analysis has utilized the device's camera to capture a pulse waveform from the finger. ${ }^{29}$ While studies that examined the diagnostic accuracy of PPG captured in this manner showed high sensitivity and specificity in differentiating AF from sinus rhythm when used in controlled environments, the primary limitations were that it could only provide a brief snapshot of the heart rate and rhythm and it put the onus on the user to consciously activate the device and remain still for the required timeframe (usually $30 \mathrm{~s}) .^{30,31}$

In contrast to the on-demand smartphone-based applications noted above, wearability allows for passive, frequent sampling of the heart rhythm using PPG and, in many cases, ECG confirmation. ${ }^{32}$ The WATCH-AF study found that a smartwatch-based PPG monitoring system had sensitivity and specificity for AF greater than $95 \%$ within a hospitalized population, which matched prior studies. ${ }^{24,33}$

A later version of the Apple Watch employed a different strategy: While the user is at rest, the watch generates a tachogram, or plot of pulse intervals, over $60 \mathrm{~s}$, and if irregularity is detected in a single tachogram, it will prompt a cascade of more frequent tachogram monitoring (Fig. 3). If five out of six consecutive tachograms are positive for irregularity, the watch will give the wearer an irregular heart rate notification. The performance of this feature was evaluated in the Apple Heart Study, which was a siteless, pragmatic, single-arm prospective observational study that enrolled over 400,000 participants over a period of 8 months. Among these participants, $2161(0.5 \%)$ were found to have an irregular 


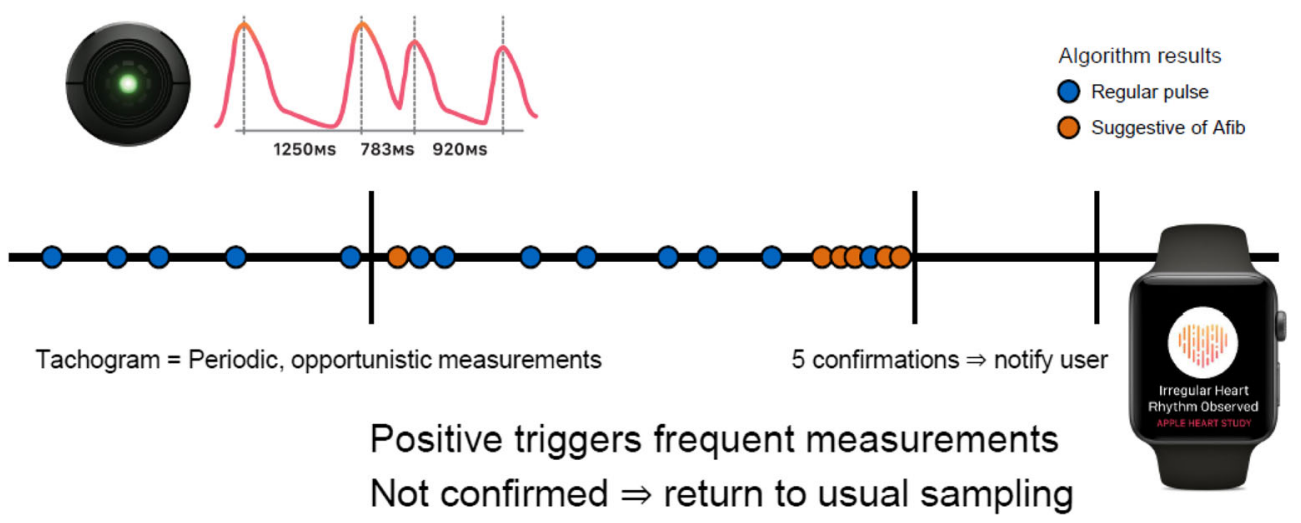

Figure 3 Schematic demonstrating the algorithm used by the early version of the Apple Watch, which was featured in the Apple Heart Study. When a user is at rest, the watch generates a 60-second tachogram. If irregularity is noted, the device will trigger subsequent tachograms; if five of six tachograms are irregular, the user receives a notification of an irregular heart rate. Importantly, more contemporary versions of the Apple Watch include both PPG (shown here) and ECG (not shown here) features.

pulse as detected by Apple Watch. In situations where an irregular rhythm was detected, participants were instructed to arrange a telehealth visit and were given an ECG patch (ePatch) by mail to be worn for at least 7 days. Of the 450 participants who returned the ePatch, 153 (34\%) were found to have confirmed AF. The study was limited by lack of simultaneous ECG confirmation, and due to the paroxysmal nature of AF, patients who may have had AF during the time of the notification by the Apple Watch may not have had recurrence while wearing the ePatch. Subsequent irregular heart rate notifications were then compared to findings on the ePatch, and the investigators reported a positive predictive value of 0.84. Another important takeaway from this study relates to early concerns that direct-to-consumer wearable devices would lead to over-detection and overutilization of the healthcare system. The Apple Heart Study found a reassuringly overall low notification rate of $0.52 \%$ that appropriately increased with age, suggesting that the algorithm, while imperfect, is unlikely to generate false positive notifications in low-risk individuals.

Importantly, PPG has limitations that must be acknowledged. First, wrist-worn devices are quite sensitive to motion artifact. ${ }^{26}$ The Apple Watch irregular notification feature addresses this by only sampling pulse intervals when it detects that the user is at rest, which impairs detection of AF during periods of exercise or arm motion. Second, device performance depends on algorithm design; for example, the Apple Watch algorithm only generates a notification if five of six tachograms meet criteria for irregularity. However, each tachogram is triggered at 15 -min intervals, meaning that this approach would miss AF episodes shorter than $75 \mathrm{~min}$, which if recurrent throughout a given day could still translate to a clinically meaningful AF burden. Third, the specificity of PPG algorithms for AF detection is impaired in the presence of another reason for an irregular pulse, such as frequent premature atrial or ventricular contractions. ${ }^{34,35}$

ECG-based algorithms provide a heart rate measurement and generally classify the patient's rhythm as sinus, AF, or inconclusive. Among commercially available smartphonebased devices, several technology companies have created products capable of producing a point-of-care ECG tracing, perhaps most notably the AliveCor Kardia Monitor device series. In a large cohort study based in Hong Kong, investigation of the Kardia single-lead ECG device found that $65 \%$ of device-detected AF were confirmed to be accurate by a reviewing cardiologist. In this study of over 10,000 patients with mean age of 78 years, the number needed to screen to make a new accurate AF diagnosis was 145 participants. ${ }^{36}$ The sensitivity and specificity of the Kardia monitor were found to be $99.6 \%$ and $97.8 \%$, respectively, in the prospective, 2-center, clinician-blinded DETECT AF PRO study. ${ }^{37}$

It is notable that the Apple Heart Study discussed above used an earlier iteration of the Apple Watch that did not have a built-in ECG function to allow for simultaneous rhythm confirmation; it only had access to PPG technology. The Apple Watch Series 4 debuted in the fall of 2018; this model was the first version of the Apple Watch with built-in single-lead ECG capability enabled by the user placing their contralateral hand on the bevel of the watch. When the watch's PPG feature produces an irregular rhythm notification, the user is prompted to obtain an ECG. In a validation study, the performance of the Apple Watch ECG and an automated app for AF detection showed a sensitivity of $98.3 \%$ and specificity of $99.6 \%$ in distinguishing AF from sinus rhythm, resulting in FDA clearance for use in those without a known AF history (https:// www.apple.com/healthcare/docs/site/Apple_Watch_Arrhythmia_Detection.pdf). Wearable devices that blend PPG and ECG features can enable constant, passive PPG monitoring of pulse irregularity and can then prompt the wearer to obtain an ECG for confirmation if irregularity is detected.

ECG capability is particularly important in allowing remote communication between patients and providers at the time of symptom onset; real-world examples are illustrated in Figure 2.

\section{DIAGNOSTIC CONFIRMATION}

Based on studies examining the accuracy of mHealth devices for facilitating AF detection noted above, diagnostic 
confirmation of AF by a physician is still needed, particularly for devices that rely solely on PPG. While medical-grade monitoring is required in devices where PPG alone is used, a device-rendered ECG strip of AF is likely sufficient to make a diagnosis of AF if confirmed by a physician. Blended technologies that utilize both PPG and ECG provide an innovative way to facilitate early AF detection, but the approach is still limited. First, charging requirements prevent uninterrupted continued use and many patients choose not to wear these devices during sleep. Second, the user must consciously activate the ECG, which could be problematic for confirming episodes if such alerts are ignored. Third, while software within these devices can read the ECG as normal rhythm or AF, these recordings can be inaccurate and it is incumbent on a medical provider to confirm these findings. Last, the technology does not currently offer information on quantifying AF burden or duration, which may be important in some scenarios.

If device-based ECG confirmation is not available, diagnostic confirmation is recommended using a conventional, continuous ECG monitor based on the frequency of episodes. While these medical-grade technologies have high accuracy and can quantify AF burden and duration, the delay between detection of AF using mHealth and application of a provider-ordered monitor may result in missed confirmation of $\mathrm{AF}$, especially if the episodes are infrequent. The use of these time-limited continuous monitors is appropriate if knowledge of a patient's AF burden and/or duration would impact clinical decision-making. Regardless, physician adjudication is needed to ensure accuracy. This can translate to a significant amount of data generated by mHealth that will require physician interpretation, although efforts to apply machine learning to this problem are ongoing. ${ }^{38}$ Table 1 presents the current FDA clearances for these devices in detecting heart rhythm irregularities Table 2.

\section{PRIMARY CARE PERSPECTIVE}

As these devices become increasingly common, it can be expected that the primary care practitioner will face questions regarding AF screening, diagnosis, and management. In light of the aforementioned uses and limitations, when is it appropriate to recommend a commercially available heart rate or rhythm monitor for $\mathrm{AF}$ screening? For a screening tool to be effective, it must be inexpensive, easy to use, and available; carry minimal risk; and be focused on an at-risk population. Furthermore, the disease itself must be serious, the prevalence must be high, and there must be data supporting the use of early intervention prior to the onset of a major adverse event. Though AF screening in a highrisk population appears to fulfill these requirements, there are no consistent recommendations on AF screening beyond the use of the physical exam and there are no specific recommendations on the use of mHealth for this purpose. ${ }^{39,40}$ Completed and ongoing studies, including the Huawei Heart Study and Heartline Study, are examining the use of these technologies for AF screening $^{41}$ (https://clinicaltrials.gov/ct2/show/ NCT04276441).

In contrast to a formalized screening program, a far more common scenario is when an asymptomatic patient with no prior history of AF presents to care with an mHealth device that suggests a new diagnosis of AF. Figure 4 proposes an algorithm for clinical response; it should be noted that the most important step is confirmation that the AF diagnosis is true. This can be done by close review of the device-rendered ECG strips, ECG at the time of the medical encounter if cardiac auscul-

Table 1 Uses for Commercially Available Ambulatory Rhythm Monitoring, with Indications for deVices Whose Use Is Cleared by the United States Food and Drug Administration (FDA)

\begin{tabular}{|c|c|c|}
\hline Uses & FDA clearance & FDA-approved devices \\
\hline Detection of atrial fibrillation & Yes & $\begin{array}{l}\text { 1. AliveCor Heart Monitor }{ }^{1} \\
\text { 2. AliveCor KardiaMobile original and } 6 \mathrm{~L}^{1,2} \\
\text { 3. AliveCor KardiaBand (accessory for Apple Watch or iPhone/iPad) } \\
\text { 4. Irregular Rhythm Notification feature on Apple Watch } \\
\text { 5. ECG app on Apple Watch } \\
\text { 6. ECG app on Fitbit Sense } \\
\text { 7. ECG Monitor app on Samsung devices, including Galaxy Watch } 3^{7} \\
\text { AliveCor KardiaMobile } 6 \mathrm{~L}^{8}\end{array}$ \\
\hline \multicolumn{3}{|c|}{$\begin{array}{l}\text { - Evaluate heart rate control in the setting of AF } \\
\text { - Assessment of rhythm control in the patient managed with anti-arrhythmics or post-AF ablation } \\
\text { - Targeted use of "pill-in-pocket" anticoagulation } \\
\text { - Evaluated use of "pill-in-pocket" anti-arrhythmic medications }\end{array}$} \\
\hline
\end{tabular}

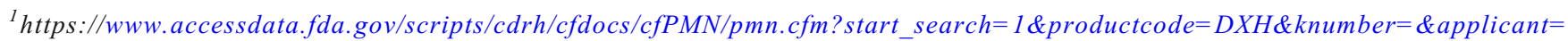
ALIVECOR\%2C\%20INC\%2E

${ }^{2}$ https://www.alivecor.com/press/press release/fda-grants-first-ever-clearance-for-six-lead-personal-ecg-device/

${ }^{3}$ https://www.accessdata.fda.gov/cdrh_docs/pdf17/K171816.pdf

${ }^{4}$ https://www.accessdata.fda.gov/cdrh docs/reviews/DEN180042.pdf

${ }^{5}$ https://www.accessdata.fda.gov/cdrh_docs/reviews/DEN180044.pdf

${ }^{6} \mathrm{https}: / / w w w$.businesswire.com/news/home/20200914005215/en/

${ }^{7}$ https://www.accessdata.fda.gov/cdrh_docs/pdf20/K201168.pdf

${ }^{8} \mathrm{https} / / / w w w$.alivecor.com/press/press_release/new-fda-guidance-allows-use-of-kardiamobile-6l-to-measure-qtc-in-covid-19-patients/ 
Table 2 Validation Studies for Selected Ambulatory Rhythm Monitoring Technologies for Atrial Fibrillation

\begin{tabular}{|c|c|c|c|c|c|c|}
\hline & Device & $n$ & Setting & Comparator & $\begin{array}{l}\text { Sensitivity } \\
(\%)\end{array}$ & $\begin{array}{l}\text { Specificity } \\
(\%)\end{array}$ \\
\hline \multirow[t]{4}{*}{ Hand-held ECG devices } & Zenicor $^{21}$ & 100 & $\begin{array}{l}\text { Outpatient cardiology } \\
\text { clinic }\end{array}$ & $\begin{array}{l}\text { 12-lead ECG } \\
\text { interpreted by }\end{array}$ & 96 & 72 \\
\hline & MyDiagnostick $^{22}$ & 192 & $\begin{array}{l}\text { Outpatient cardiology } \\
\text { clinic }\end{array}$ & $\begin{array}{l}\text { 12-lead ECG } \\
\text { interpreted by } \\
\text { cardiologist }\end{array}$ & 100 & 96 \\
\hline & Omron HCG- $801^{23}$ & 999 & Primary care practices & $\begin{array}{l}\text { 12-lead ECG } \\
\text { interpreted by } \\
\text { cardiologist }\end{array}$ & 94.4 & 94.6 \\
\hline & $\begin{array}{l}\text { Merlin ECG event } \\
\text { recorders }\end{array}$ & 999 & Primary care practices & $\begin{array}{l}\text { 12-lead ECG } \\
\text { interpreted by }\end{array}$ & 93.9 & 90.1 \\
\hline PPG & Apple Watch ${ }^{24}$ & 51 & $\begin{array}{l}\text { Recruited patients } \\
\text { undergoing cardioversion }\end{array}$ & $\begin{array}{l}\text { 12-lead ECG } \\
\text { interpreted by } \\
\text { cardiologist }\end{array}$ & 98.0 & 90.2 \\
\hline Smartphone ECG device & $\begin{array}{l}\text { AliveCor Kardia } \\
\text { Mobile }^{25}\end{array}$ & 204 & Recruited patients & $\begin{array}{l}\text { 12-lead ECG } \\
\text { interpreted by } \\
\text { cardiologist }\end{array}$ & 98 & 97 \\
\hline $\begin{array}{l}\text { Smartwatch with combined } \\
\text { ECG and PPG features }\end{array}$ & $\begin{array}{l}\text { Samsung Simband } \\
2.0^{26}\end{array}$ & 40 & $\begin{array}{l}\text { Recruited patients from } \\
\text { outpatient clinic }\end{array}$ & Holter monitor & 98.2 & 98.1 \\
\hline
\end{tabular}

tation is suggestive of AF, or follow-up with ambulatory rhythm monitoring, such as those described above. Subclinical $\mathrm{AF}$ as detected by implantable cardiac devices, such as pacemakers and defibrillators, has previously been shown to be associated with increased rates of stroke; thus, by extrapolating this data, it would be reasonable to consider therapy to reduce thromboembolic stroke risk in those found to have mHealth-detected AF and with associated risk factors, per $\mathrm{CHA}_{2} \mathrm{DS}_{2}$-VASc scoring., $52-45$

\section{BILLING FOR MONITORING TECHNOLOGY AND INCORPORATING PATIENT MONITORING DATA INTO EHR}

The increased integration of mHealth into screening and early identification of patients at risk of AF has significant implications for reimbursement and overall billing strategies. The European Health Economic Trial on Home Monitoring in ICD Therapy (EuroEco) suggested that follow-up costs for

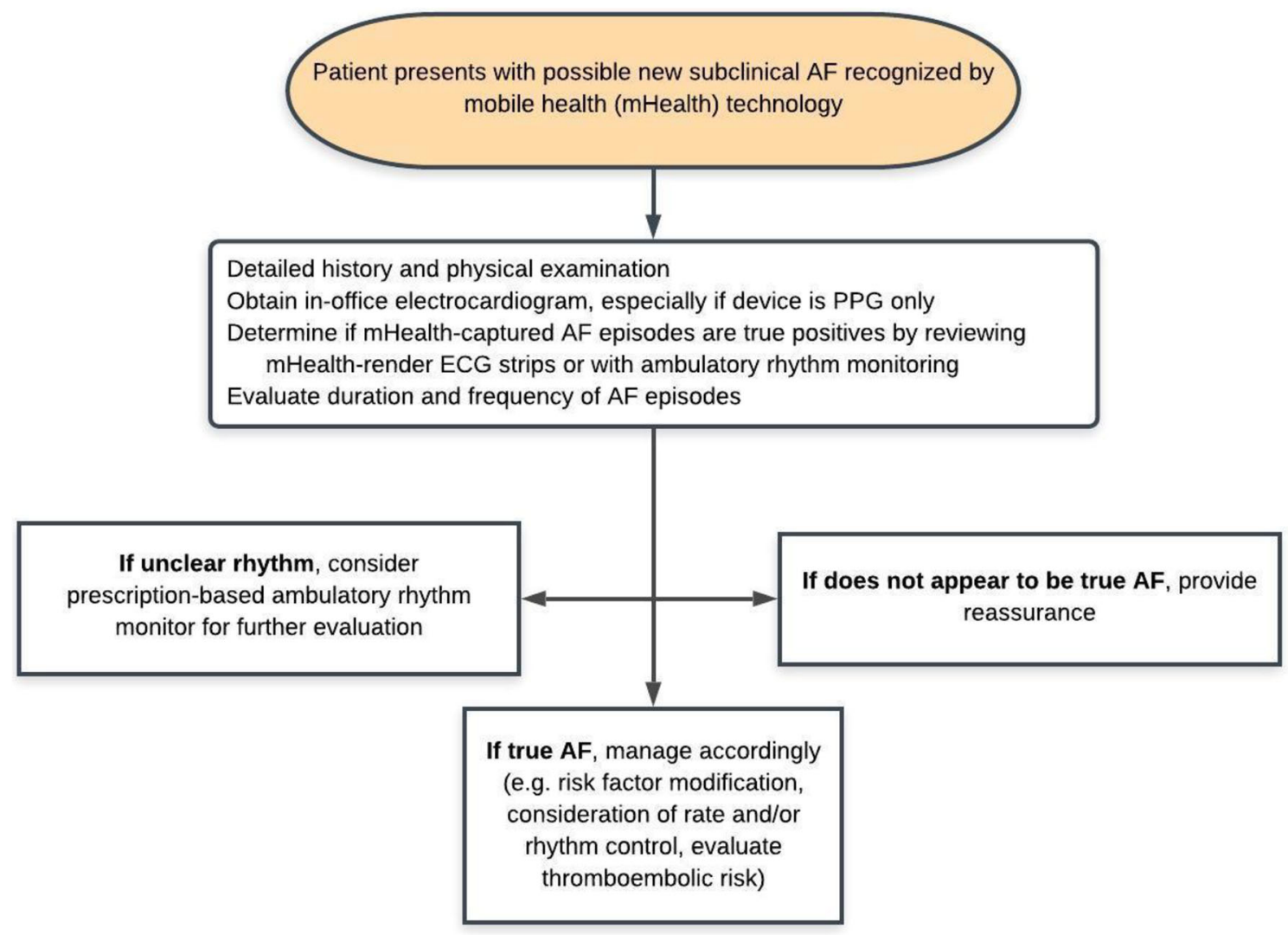

Figure 4 Proposed management schema for the management of new subclinical AF as recognized by mobile health technology. 
providers were similar, regardless of whether the patient was seen remotely or in clinic. Despite the necessary structural reorganization to accommodate remote visits, the cost of healthcare both for the payers and for the providers was statistically similar. Additionally, the study found that participants monitoring their symptoms from home had numerically fewer hospitalizations and shorter length of stay. ${ }^{46}$

As healthcare, particularly in the space of arrhythmia management, is increasingly delivered remotely, there is a clear need to revise the billing system. The conventional billing strategy for discrete events proves less favorable. Instead, one group suggested a subscription-based system that gives patients a 1-year access to hardware, software, and other services to monitor and analyze continuous input from devices. This strategy would compensate providers for caring for patients remotely and enable patients to continue receiving accurate and timely interpretation of results. ${ }^{47}$

In addition to billing, an emerging challenge is incorporation of an increasing burden of remote data into a patient's electronic health records. Provider experience with CIEDs could shed insight into this transition. CIEDs currently transmit data from a single patient to a remote transceiver and ultimately the manufacturer's server. Individual practices are able to access the server in order to analyze the patient data, and these data reports can be uploaded into a patient's chart. Unfortunately, many current EHR systems do not permit patients to send attachments; as a result, patients often use non-HIPAA-compliant means of communication, including email, to share personal health information. One potential solution would be a secure cloud-based design to store patient data and for providers to them access directly.

\section{LEGAL CONSIDERATIONS FOR PHYSICIANS USING SCREENING PROGRAMS}

While monitoring patients can potentially detect latent cardiovascular risk factors, there are several legal responsibilities that must be addressed to ensure that patients and providers are providing cost-effective and safe care. Since monitoring technologies are becoming increasingly accessible to the masses, the focus is shifting from physicians administering screening tests to patients individually sharing personal health data with their medical teams. As a result, physicians must balance this increasing influx of data with efficient data review and effective follow-up. While the traditional medicolegal framework has not been adapted for managing this new source of patient-driven data, it is recommended that providers adhere to clear practices to reduce the risk of litigation and improve patient outcomes: clear communication with patients, timely follow-up with data, and clear counseling. ${ }^{48}$ The medicolegal ramifications of a missed diagnosis of AF that was captured on mHealth or, yet worse, a cardioembolic stroke that occurred while off anticoagulation in a patient in whom AF was detected on mHealth are unclear at this point.

\section{A PROMISING FUTURE: OPPORTUNITIES BEYOND AF DETECTION}

While it is clear that increased AF burden is associated with higher risk of systemic embolism, most of the prior studies establishing this pattern were performed in patients with cardiac implantable electronic devices (CIEDs), rather than commercially available mHealth technologies. ${ }^{34,42-44,49-52}$ The clinical implications for mHealth-detected AF, as compared to conventionally detected AF, are not yet known. Other theoretical uses for mHealth include targeted, or "pill-in-pocket," anticoagulation ${ }^{53,54}$; QTc monitoring in patients taking QT-prolonging medications ${ }^{55}$; on-demand rhythm control with pill-in-pocket anti-arrhythmic medications ${ }^{56}$; and evaluation of long-term rate control in AF. At this current time, these uses are largely investigative, except for those outlined in Table 1. The possibilities in this space are incredible, but further rigorous investigation is needed to clarify their clinical utility.

\section{CONCLUSIONS}

Commercially available mHealth technologies are becomingly increasingly more common, and clinicians now face the challenge of interpreting their data and deciding how to incorporate these data into a particular patient's medical profile. There are many possible uses for these data, including evaluation of on-demand ECG strips at time of palpitations and screening for subclinical AF; however, incorporation of these data into clinical decision-making models is in its relative infancy. Further studies are needed to define how to use these data in an evidence-based manner.

Corresponding Author: Jayson R. Baman, MD; Division of Cardiology, Department of Medicine, Northwestern University Feinberg School of Medicine, Chicago, IL, USA (e-mail: jayson. baman@northwestern.edu).

\section{Declarations:}

Conflict of Interest: Dr. Passman is on the advisory board of Medtronic, receives research grant funding from Abbott, and receives royalties from UpToDate. Dr. Baman, Mr. Mathew, and Dr. Jiang report no disclosures.

\section{REFERENCES}

1. Wolf PA, Dawber TR, Thomas HE, Jr., Kannel WB. Epidemiologic assessment of chronic atrial fibrillation and risk of stroke: the Framingham study. Neurology. 1978;28(10):973-7. doi:https://doi.org/10.1212/ wnl.28.10.973

2. Bakhai A, Darius H, De Caterina R, Smart A, Le Heuzey JY, Schilling RJ, et al. Characteristics and outcomes of atrial fibrillation patients with or without specific symptoms: results from the PREFER in AF registry. Eur Heart J Qual Care Clin Outcomes. 2016;2(4):299-305. doi:https://doi. org/10.1093/ehjqcco/qcw031

3. January CT, Wann LS, Alpert JS, Calkins H, Cigarroa JE, Cleveland JC, Jr., et al. 2014 AHA/ACC/HRS guideline for the management of patients with atrial fibrillation: a report of the American College of Cardiology/ American Heart Association Task Force on Practice Guidelines and the 
Heart Rhythm Society. J Am Coll Cardiol. 2014;64(21):e1-76. doi:https://doi.org/10.1016/j.jacc.2014.03.022

4. Benjamin EJ, Wolf PA, D’Agostino RB, Silbershatz H, Kannel WB, Levy D. Impact of atrial fibrillation on the risk of death: the Framingham Heart Study. Circulation. 1998;98(10):946-52. doi:https://doi.org/10.1161/ 01.cir.98.10.946

5. Freedman B, Camm J, Calkins H, Healey JS, Rosenqvist M, Wang J, et al. Screening for Atrial Fibrillation: A Report of the AF-SCREEN International Collaboration. Circulation. 2017;135(19):1851-67. doi:https://doi. org/10.1161/CIRCULATIONAHA. 116.026693

6. Passman R, Bernstein RA. New Appraisal of Atrial Fibrillation Burden and Stroke Prevention. Stroke. 2016;47(2):570-6. doi:https://doi.org/10. 1161/STROKEAHA.115.009930

7. Friberg L, Rosenqvist M, Lindgren A, Terent A, Norrving B, Asplund K. High prevalence of atrial fibrillation among patients with ischemic stroke. Stroke. 2014;45(9):2599-605. doi:https://doi.org/10.1161/ STROKEAHA.114.006070

8. Mittal S, Movsowitz C, Steinberg JS. Ambulatory external electrocardiographic monitoring: focus on atrial fibrillation. J Am Coll Cardiol. 2011;58(17):1741-9. doi:https://doi.org/10.1016/j.jacc.2011.07.026

9. Thavendiranathan P, Bagai A, Khoo C, Dorian P, Choudhry NK. Does this patient with palpitations have a cardiac arrhythmia? JAMA. 2009;302(19):2135-43. doi:https://doi.org/10.1001/jama.2009.1673

10. Probst MA, Mower WR, Kanzaria HK, Hoffman JR, Buch EF, Sun BC. Analysis of emergency department visits for palpitations (from the National Hospital Ambulatory Medical Care Survey). Am J Cardiol. 2014;113(10):1685-90. doi:https://doi.org/10.1016/j.amjcard.2014.02. 020

11. Rothman SA, Laughlin JC, Seltzer J, Walia JS, Baman RI, Siouffi SY, et al. The diagnosis of cardiac arrhythmias: a prospective multi-center randomized study comparing mobile cardiac outpatient telemetry versus standard loop event monitoring. J Cardiovasc Electrophysiol. 2007;18(3):241-7. doi:https://doi.org/10.1111/j.1540-8167.2006. 00729.x

12. Sulfi S, Balami D, Sekhri N, Suliman A, Kapur A, Archbold RA, et al. Limited clinical utility of Holter monitoring in patients with palpitations or altered consciousness: analysis of 8973 recordings in 7394 patients. Ann Noninvasive Electrocardiol. 2008;13(1):39-43. doi:https://doi.org/10. $1111 /$ j. 1542-474X.2007.00199.x

13. Giada F, Gulizia M, Francese M, Croci F, Santangelo L, Santomauro M, et al. Recurrent unexplained palpitations (RUP) study comparison of implantable loop recorder versus conventional diagnostic strategy. J Am Coll Cardiol. 2007;49(19):1951-6. doi:https://doi.org/10.1016/j.jacc. 2007.02.036

14. Paudel B, Paudel K. The diagnostic significance of the holter monitoring in the evaluation of palpitation. Journal of clinical and diagnostic research : JCDR. 2013;7(3):480-3. doi:https://doi.org/10.7860/JCDR/ 2013/4923.2802

15. Sanna T, Diener HC, Passman RS, Di Lazzaro V, Bernstein RA, Morillo $\mathrm{CA}$, et al. Cryptogenic stroke and underlying atrial fibrillation. N Engl J Med. 2014;370(26):2478-86. doi:https://doi.org/10.1056/ NEJMoa 1313600

16. Choe WC, Passman RS, Brachmann J, Morillo CA, Sanna T, Bernstein RA, et al. A Comparison of Atrial Fibrillation Monitoring Strategies After Cryptogenic Stroke (from the Cryptogenic Stroke and Underlying AF Trial). Am J Cardiol. 2015;116(6):889-93. doi:https://doi.org/10.1016/j. amjcard.2015.06.012

17. Yano Y, Greenland P, Lloyd-Jones DM, Daoud EG, Koehler JL, Ziegler PD. Simulation of Daily Snapshot Rhythm Monitoring to Identify Atrial Fibrillation in Continuously Monitored Patients with Stroke Risk Factors. PLoS One. 2016;11(2):e0148914. doi:https://doi.org/10.1371/journal. pone.0148914

18. Lohrmann G, Kaplan R, Ziegler PD, Monteiro J, Passman R. Atrial Fibrillation Ablation Success Defined by Duration of Recurrence on Cardiac Implantable Electronic Devices. J Cardiovasc Electrophysiol. 2020. doi:https://doi.org/10.1111/jce.14781

19. Gladstone DJ, Spring M, Dorian P, Panzov V, Thorpe KE, Hall J, et al. Atrial fibrillation in patients with cryptogenic stroke. N Engl J Med. 2014;370(26):2467-77. doi:https://doi.org/10.1056/NEJMoa1311376

20. McConnell MV, Turakhia MP, Harrington RA, King AC, Ashley EA. Mobile Health Advances in Physical Activity, Fitness, and Atrial Fibrillation: Moving Hearts. J Am Coll Cardiol. 2018;71(23):2691-701. doi:https:// doi.org/10.1016/j.jacc.2018.04.030

21. Doliwa PS, Frykman V, Rosenqvist M. Short-term ECG for out of hospital detection of silent atrial fibrillation episodes. Scand Cardiovasc J. 2009;43(3):163-8. doi:https://doi.org/10.1080/14017430802593435
22. Tieleman RG, Plantinga Y, Rinkes D, Bartels GL, Posma JL, Cator R, et al. Validation and clinical use of a novel diagnostic device for screening of atrial fibrillation. Europace. 2014;16(9):1291-5. doi:https://doi.org/10. 1093/europace/euu057

23. Kearley K, Selwood M, Van den Bruel A, Thompson M, Mant D, Hobbs FR, et al. Triage tests for identifying atrial fibrillation in primary care: a diagnostic accuracy study comparing single-lead ECG and modified BP monitors. BMJ Open. 2014;4(5):e004565. doi:https://doi.org/10.1136/ bmjopen-2013-004565

24. Tison GH, Sanchez JM, Ballinger B, Singh A, Olgin JE, Pletcher MJ, et al. Passive Detection of Atrial Fibrillation Using a Commercially Available Smartwatch. JAMA Cardiol. 2018;3(5):409-16. doi:https://doi.org/10. 1001/jamacardio.2018.0136

25. Lau JK, Lowres N, Neubeck L, Brieger DB, Sy RW, Galloway CD, et al. iPhone ECG application for community screening to detect silent atrial fibrillation: a novel technology to prevent stroke. Int $\mathrm{J}$ Cardiol. 2013;165(1): 193-4. doi:https://doi.org/10.1016/j.ijcard.2013.01.220

26. Ding EY, Han D, Whitcomb C, Bashar SK, Adaramola O, Soni A, et al. Accuracy and Usability of a Novel Algorithm for Detection of Irregular Pulse Using a Smartwatch Among Older Adults: Observational Study. JMIR Cardio. 2019;3(1):e13850. doi:https://doi.org/10.2196/13850

27. Pereira T, Tran N, Gadhoumi K, Pelter MM, Do DH, Lee RJ, et al. Photoplethysmography based atrial fibrillation detection: a review. NPJ Digit Med. 2020;3:3. doi:https://doi.org/10.1038/s41746-019-0207-9

28. Pipitprapat W, Harnchoowong S, Suchonwanit P, Sriphrapradang C. The validation of smartphone applications for heart rate measurement. Ann Med. 2018;50(8):721-7. doi:https://doi.org/10.1080/07853890.2018. 1531144

29. Proesmans T, Mortelmans C, Van Haelst R, Verbrugge F, Vandervoort P, Vaes B. Mobile Phone-Based Use of the Photoplethysmography Technique to Detect Atrial Fibrillation in Primary Care: Diagnostic Accuracy Study of the FibriCheck App. JMIR Mhealth Uhealth. 2019;7(3):e12284. doi:https://doi.org/10.2196/12284

30. O’Sullivan JW, Grigg S, Crawford W, Turakhia MP, Perez M, Ingelsson E, et al. Accuracy of Smartphone Camera Applications for Detecting Atrial Fibrillation: A Systematic Review and Meta-analysis. JAMA Netw Open. 2020;3(4):e202064. doi:https://doi.org/10.1001/jamanetworkopen. 2020.2064

31. McManus DD, Lee J, Maitas O, Esa N, Pidikiti R, Carlucci A, et al. A novel application for the detection of an irregular pulse using an iPhone $4 \mathrm{~S}$ in patients with atrial fibrillation. Heart Rhythm. 2013;10(3):315-9. doi:https://doi.org/10.1016/j.hrthm.2012.12.001

32. Ip JE. Wearable Devices for Cardiac Rhythm Diagnosis and Management. JAMA. 2019;321(4):337-8. doi:https://doi.org/10.1001/jama.2018. 20437

33. Dorr M, Nohturfft V, Brasier N, Bosshard E, Djurdjevic A, Gross S, et al. The WATCH AF Trial: SmartWATCHes for Detection of Atrial Fibrillation. JACC Clin Electrophysiol. 2019;5(2):199-208. doi:https://doi.org/10. 1016/j.jacep.2018.10.006

34. Wasserlauf J, You C, Patel R, Valys A, Albert D, Passman R. Smartwatch Performance for the Detection and Quantification of Atrial Fibrillation. Circ Arrhythm Electrophysiol. 2019;12(6):e006834. doi:https://doi.org/ 10.1161/CIRCEP.118.006834

35. Perez MV, Mahaffey KW, Hedlin H, Rumsfeld JS, Garcia A, Ferris T, et al. Large-Scale Assessment of a Smartwatch to Identify Atrial Fibrillation. N Engl J Med. 2019;381(20):1909-17. doi:https://doi.org/10.1056/ NEJMoa1901183

36. Chan NY, Choy CC, Chan CK, Siu CW. Effectiveness of a nongovernmental organization-led large-scale community atrial fibrillation screening program using the smartphone electrocardiogram: An observational cohort study. Heart Rhythm. 2018;15(9):1306-11. doi:https://doi.org/ 10.1016/j.hrthm.2018.06.006

37. Brasier N, Raichle CJ, Dorr M, Becke A, Nohturfft V, Weber S, et al. Detection of atrial fibrillation with a smartphone camera: first prospective, international, two-centre, clinical validation study (DETECT AF PRO). Europace. 2019;21(1):41-7. doi:https://doi.org/10.1093/ europace/euy176

38. Hannun AY, Rajpurkar P, Haghpanahi M, Tison GH, Bourn C, Turakhia MP, et al. Cardiologist-level arrhythmia detection and classification in ambulatory electrocardiograms using a deep neural network. Nat Med. 2019;25(1):65-9. doi:https://doi.org/10.1038/s41591-018-0268-3

39. Hindricks G, Potpara T, Dagres N, Arbelo E, Bax JJ, BlomstromLundqvist $\mathrm{C}$, et al. 2020 ESC Guidelines for the diagnosis and management of atrial fibrillation developed in collaboration with the European Association of Cardio-Thoracic Surgery (EACTS). Eur Heart J. 2020. doi:https://doi.org/10.1093/eurheartj/ehaa612 
40. January CT, Wann LS, Calkins H, Chen LY, Cigarroa JE, Cleveland JC, Jr., et al. 2019 AHA/ACC/HRS Focused Update of the 2014 AHA/ACC/ HRS Guideline for the Management of Patients With Atrial Fibrillation: A Report of the American College of Cardiology/American Heart Association Task Force on Clinical Practice Guidelines and the Heart Rhythm Society in Collaboration With the Society of Thoracic Surgeons. Circulation. 2019;140(2):e125-e51. doi:https://doi.org/10.1161/CIR. 0000000000000665

41. Guo $Y$, Wang $H$, Zhang $H$, Liu $T$, Liang $Z$, Xia $Y$, et al. Mobile Photoplethysmographic Technology to Detect Atrial Fibrillation. J Am Coll Cardiol. 2019;74(19):2365-75. doi:https://doi.org/10.1016/j.jacc. 2019.08.019

42. Glotzer TV, Daoud EG, Wyse DG, Singer DE, Holbrook R, Pruett K, et al. Rationale and design of a prospective study of the clinical significance of atrial arrhythmias detected by implanted device diagnostics: the TRENDS study. Journal of interventional cardiac electrophysiology : an international journal of arrhythmias and pacing. 2006;15(1):9-14. doi:https:// doi.org/10.1007/s10840-006-7622-y

43. Glotzer TV, Daoud EG, Wyse DG, Singer DE, Ezekowitz MD, Hilker C, et al. The relationship between daily atrial tachyarrhythmia burden from implantable device diagnostics and stroke risk: the TRENDS study. Circ Arrhythm Electrophysiol. 2009;2(5):474-80. doi:https://doi.org/10. 1161/CIRCEP. 109.849638

44. Capucci A, Santini M, Padeletti L, Gulizia M, Botto G, Boriani G, et al. Monitored atrial fibrillation duration predicts arterial embolic events in patients suffering from bradycardia and atrial fibrillation implanted with antitachycardia pacemakers. J Am Coll Cardiol. 2005;46(10):1913-20. doi:https://doi.org/10.1016/j.jacc.2005.07.044

45. Botto GL, Padeletti L, Santini M, Capucci A, Gulizia M, Zolezzi F, et al. Presence and duration of atrial fibrillation detected by continuous monitoring: crucial implications for the risk of thromboembolic events. J Cardiovasc Electrophysiol. 2009;20(3):241-8. doi:https://doi.org/10. $1111 / \mathrm{j} .1540-8167.2008 .01320 . x$

46. Heidbuchel H, Hindricks G, Broadhurst P, Van Erven L, FernandezLozano I, Rivero-Ayerza M, et al. EuroEco (European Health Economic Trial on Home Monitoring in ICD Patients): a provider perspective in five European countries on costs and net financial impact of follow-up with or without remote monitoring. Eur Heart J. 2015;36(3):158-69. doi:https:// doi.org/10.1093/eurheartj/ehu339

47. Jiang X, Ming WK, You JH. The Cost-Effectiveness of Digital Health Interventions on the Management of Cardiovascular Diseases: Systematic Review. J Med Internet Res. 2019;21(6):e13166. doi:https://doi.org/10. 2196/13166

48. Orchard JJ, Neubeck L, Orchard JW, Puranik R, Raju H, Freedman B, et al. ECG-based cardiac screening programs: Legal, ethical, and logistical considerations. Heart Rhythm. 2019;16(10):1584-91. doi:https://doi.org/10.1016/j.hrthm.2019.03.025
49. Kaplan RM, Koehler J, Ziegler PD, Sarkar S, Zweibel S, Passman RS. Stroke Risk as a Function of Atrial Fibrillation Duration and CHA2DS2VASc Score. Circulation. 2019;140(20):1639-46. doi:https://doi.org/10. 1161/CIRCULATIONAHA.119.041303

50. Kirchhof P, Blank BF, Calvert M, Camm AJ, Chlouverakis G, Diener HC, et al. Probing oral anticoagulation in patients with atrial high rate episodes: Rationale and design of the Non-vitamin $\mathrm{K}$ antagonist Oral anticoagulants in patients with Atrial High rate episodes (NOAH-AFNET 6) trial. American heart journal. 2017;190:12-8. doi:https://doi.org/10. 1016/j.ahj.2017.04.015

51. Lopes RD, Alings M, Connolly SJ, Beresh H, Granger CB, Mazuecos JB, et al. Rationale and design of the Apixaban for the Reduction of ThromboEmbolism in Patients With Device-Detected Sub-Clinical Atrial Fibrillation (ARTESiA) trial. American heart journal. 2017;189:137-45. doi:https://doi.org/10.1016/j.ahj.2017.04.008

52. Van Gelder IC, Healey JS, Crijns H, Wang J, Hohnloser SH, Gold MR, et al. Duration of device-detected subclinical atrial fibrillation and occurrence of stroke in ASSERT. Eur Heart J. 2017;38(17):1339-44. doi:https://doi.org/10.1093/eurheartj/ehx042

53. Passman R, Leong-Sit P, Andrei AC, Huskin A, Tomson TT, Bernstein R, et al. Targeted Anticoagulation for Atrial Fibrillation Guided by Continuous Rhythm Assessment With an Insertable Cardiac Monitor: The Rhythm Evaluation for Anticoagulation With Continuous Monitoring (REACT.COM) Pilot Study. J Cardiovasc Electrophysiol. 2016;27(3):26470. doi:https://doi.org/10.1111/jce.12864

54. Waks JW, Passman RS, Matos J, Reynolds M, Thosani A, Mela T, et al Intermittent anticoagulation guided by continuous atrial fibrillation burden monitoring using dual-chamber pacemakers and implantable cardioverter-defibrillators: Results from the Tailored Anticoagulation for Non-Continuous Atrial Fibrillation (TACTIC-AF) pilot study. Heart Rhythm. 2018;15(11):1601-7. doi:https://doi.org/10.1016/j.hrthm. 2018.06.027

55. Garabelli P, Stavrakis S, Albert M, Koomson E, Parwani P, Chohan J, et al. Comparison of QT Interval Readings in Normal Sinus Rhythm Between a Smartphone Heart Monitor and a 12-Lead ECG for Healthy Volunteers and Inpatients Receiving Sotalol or Dofetilide. J Cardiovasc Electrophysiol. 2016;27(7):827-32. doi:https://doi.org/10.1111/jce. 12976

56. Alboni P, Botto GL, Baldi N, Luzi M, Russo V, Gianfranchi L, et al. Outpatient treatment of recent-onset atrial fibrillation with the "pill-inthe-pocket" approach. N Engl J Med. 2004;351(23):2384-91. doi:https:// doi.org/10.1056/NEJMoa041233

Publisher's Note: Springer Nature remains neutral with regard to jurisdictional claims in published maps and institutional affiliations. 